\title{
Summary of gene mutations outcomes in parasitic disease
}

\section{Editorial Sherif M. Abaza}

Parasitology Department, Faculty of Medicine, Suez Canal University, Ismailia, Egypt

Gene mutation may occur either in the parasite or in the host, which may be beneficial or harmful for each. In the era of parasitic diseases, the outcome of gene mutations includes 1) disease susceptibility and resistance, 2) drug resistance, 3) parasite virulence, 4) pathogenesis and host clinical presentations, 5) occurrence of malignancy, and 6) vector control. These categories were discussed in details in the previous issues [PUJ 2016; 9(2) and PUJ 2017; 10(1\&2)], whereas objective of the following editorial is to summarize and tabulate all gene mutations incriminated in the outcome of the parasitic diseases.

Key Words: Gene mutations, parasites.

Received: 12 January 2018, Accepted: 5 February 2018.

Corresponding Author: Sherif M. Abaza, Tel. : +20-10-0524-3428, E-mail: smabaza@hotmail.com.

ISSN: 1687-7942, Vol. 11, No. 1, April 2018.

Summary of host gene mutations and effect on parasitic diseases

\begin{tabular}{|c|c|c|}
\hline Gene & Effect on parasitic diseases & Reference \\
\hline actc1 & Increased susceptibility to cardiomyopathy in Chagas' disease & 122 \\
\hline $\operatorname{adam12}$ & Susceptibility to Chagas' disease & 79 \\
\hline ank-1 (Ankyrin-1) & Susceptibility to malaria & 52 \\
\hline apobec $3 b$ & Susceptibility to $P$. falciparum & 56 \\
\hline apoL-I & Susceptibility to African trypanosomiasis & 77 \\
\hline bat1 & Protection against severe malignant malaria & 114 \\
\hline ccr5 & Increased susceptibility to cardiomyopathy in Chagas' disease & 120 \\
\hline CD32 encoding gene & Susceptibility to severe malignant malaria & 113 \\
\hline CD54 encoding gene & Increased severity of falciparum malaria & 99 \\
\hline CD234 encoding gene $(f y)$ & Resistance to vivax malaria & 34 \\
\hline cdkn2 & Susceptibility to cancer bladder in urinary schistosomiasis & 83,84 \\
\hline chit & Resistance to malaria & 40 \\
\hline Cytochrome P450 encoding gene & Resistance to artemisinin therapy & 136 \\
\hline fas in mice & Susceptibility to cutaneous leishmaniasis & 67 \\
\hline ftt \& \& foxc 2 & Increased susceptibility of lymphatic filariasis & 119 \\
\hline \multirow{2}{*}{ G6PD encoding gene } & Resistance to vivax malaria & 44 \\
\hline & Protection against severe malaria & $105-109$ \\
\hline $\begin{array}{l}\text { Galactose binding protein (PpGalec) } \\
\text { encoding gene (transgenic mutation) }\end{array}$ & $\begin{array}{l}\text { Decreased infection of sandflies } \\
\text { with Leishmania spp. }\end{array}$ & 135 \\
\hline Glycophorins encoding gene & Resistance to malaria & 39 \\
\hline $\begin{array}{l}\text { Hemoglobin mutant variants (sickle } \\
\text { cell anemia \& thalassemia) }\end{array}$ & Resistance to malaria & $41-43$ \\
\hline ICAM-1 encoding gene & Increased severity of falciparum malaria & 99 \\
\hline IFN- $\gamma$ receptor encoding gene & Increased susceptibility of peri-portal fibrosis in schistosomiasis & 117,118 \\
\hline$i k b l$ & Increased susceptibility to cardiomyopathy in Chagas' disease & 121 \\
\hline$i l 2 r a$ & Susceptibility to cutaneous leishmaniasis & 70 \\
\hline $\begin{array}{l}\text { Kdr in A. gambiae \& A. funestus } \\
\text { (transgenic mutation) }\end{array}$ & $\begin{array}{l}\text { Increased resistance to DDT insecticide with } \\
\text { Increased susceptibility to } P \text {. falciparum }\end{array}$ & $124-130$ \\
\hline kras & Susceptibility to cancer bladder in urinary schistosomiasis & 88 \\
\hline Personal non-commercial use only. PUJ co & All rights reserved & 18.2622 .1009 \\
\hline
\end{tabular}


PARASITOLOGISTS UNITED JOURNAL

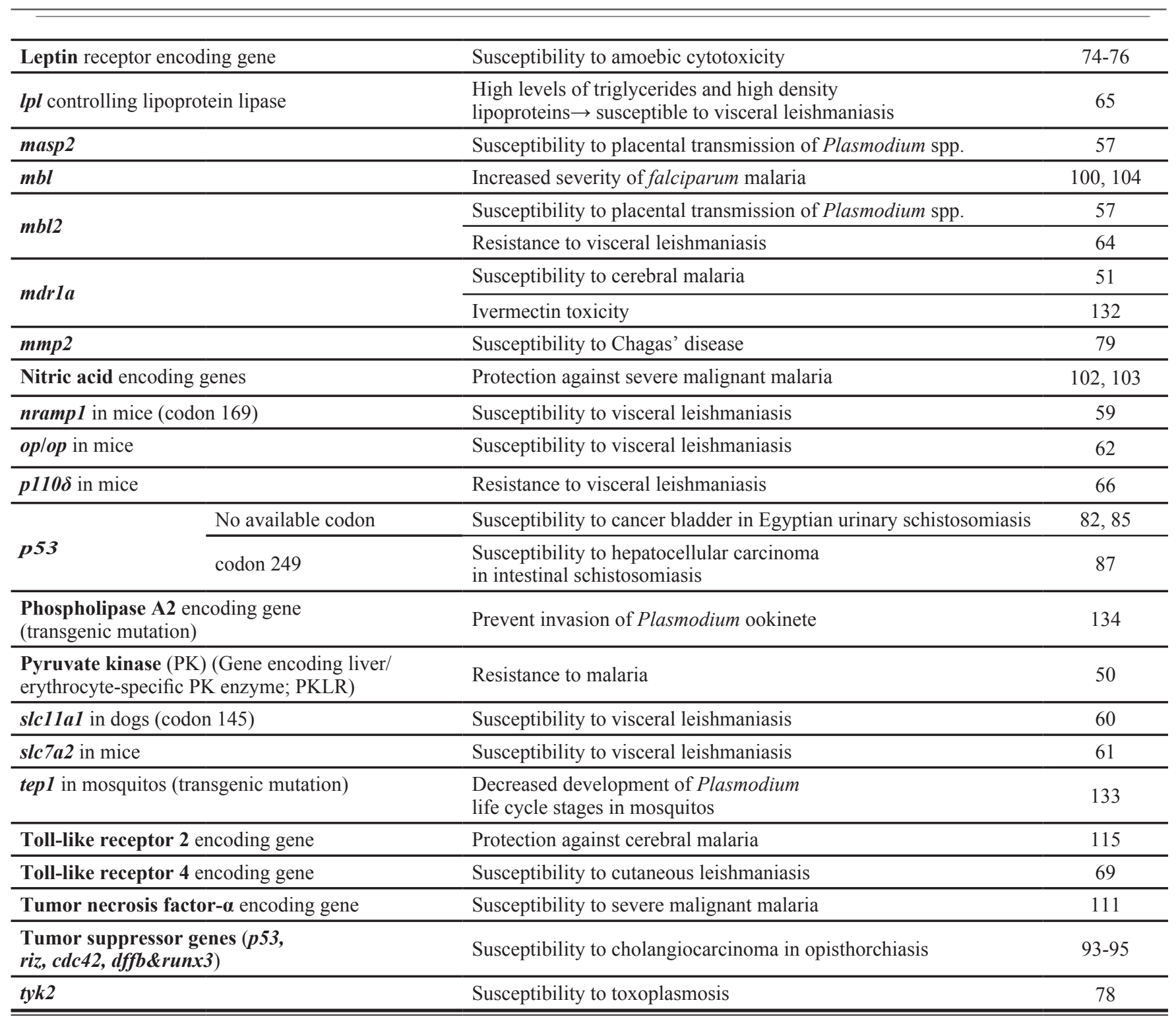

Gene abbreviations: actc1: Gene encoding cardiac muscle alpha actin; adam12: Gene encoding placental expression enzymes; ank-1: Gene encoding ankyrin-1; apobec3b: Host gene involved in innate response; apoL-I: Gene controlling high-density lipoproteins, bat1: Gene regulating cytokines production; ccr5: Gene encoding chemokine (C-C motif) receptor 5; cdkn2: Tumor suppressor gene; chit: Gene controlling chitotriosidase; fas: Apoptosis gene; flt4: Gene encoding tyrosine kinase 4 receptor, foxc2: Gene encoding fork-head box protein C2 (Transcription factor); ICAM-1: Gene encoding intercellular adhesion molecule-1; il2ra: Gene controlling IL-2 pathway; kras: Tumor suppressor gene; lpl Gene controlling lipoprotein lipase; masp2: Gene encoding mannan-binding lectin serine peptidase 2; $\boldsymbol{m b l}$ : Gene encoding mannose-binding lectin protein; $\boldsymbol{m d r 1 a}$ : Gene encoding multi-drug resistance-1a; $\boldsymbol{m m p 2 :}$ Gene encoding placental expression enzymes; $\boldsymbol{n} \boldsymbol{r a m p 1}$ : Gene controlling innate immunity against intracellular parasites; op/op: Gene controlling macrophage colony stimulating factor, p110d: Gene controlling hepatic stellate cells; p53: Tumor suppressor gene; slc11a1: Gene controlling parasite replication in the macrophage; slc7a2: Gene controlling arginine uptake by macrophages; tep1: Gene controlling immune response in mosquitos; tyk2: Gene encoding tyrosine kinase-2.

References are cited in "Gene mutations and parasitic diseases: Part I: Host gene mutations (PUJ 2016; 9: 65-79)". 
Summary of parasite gene mutations and effect on parasitic diseases

\begin{tabular}{|c|c|c|c|}
\hline \multicolumn{2}{|c|}{ Gene } & Effect on parasitic diseases & Reference \\
\hline \multicolumn{4}{|c|}{ Single gene mutation } \\
\hline \multirow{2}{*}{\multicolumn{2}{|c|}{$a q p 2$}} & Melarsoprol/pentamidine resistance for T. brucei & 181 \\
\hline & & Antimonial drugs resistance for Leishmania spp. & 193 \\
\hline \multirow{2}{*}{\multicolumn{2}{|c|}{ bat1 }} & Melarsoprol resistance for T. gambiense & 177,179 \\
\hline & & Diminazene aceturate resistance for $T$. gambiense & 180 \\
\hline \multicolumn{2}{|c|}{ B-tubulin isotype-1 gene (Codons 167,198$)$} & Benzimidazole resistance for $A$. caninum in dogs & 198 \\
\hline \multicolumn{2}{|c|}{$\beta$-tubulin isotype-2 gene } & Benzimidazole resistant for E. granulosus in human & 199 \\
\hline \multicolumn{2}{|c|}{$\operatorname{cg} 2$} & Chloroquine resistance of $P$. falciparumin vitro & 129 \\
\hline \multirow{4}{*}{ crt } & \multirow[t]{2}{*}{ Codon 76} & $\begin{array}{l}\text { Chloroquine resistance for } P \text {. falciparum in } \\
\text { Brazil and several countries in Africa \& Asia }\end{array}$ & $\begin{array}{c}96 \& \\
100-107\end{array}$ \\
\hline & & Increased severity of falciparum malaria & 209 \\
\hline & Codons $72-76$ & Amodiaquine resistance in vivo & 109 \\
\hline & Codons $76,271,326 \& 371$ & Chloroquine resistance for $P$. falciparum in Yemen & 111 \\
\hline \multirow{2}{*}{$c y b$} & \multirow{2}{*}{ No available codons } & Antimycin A resistance for Leishmania spp. & 189 \\
\hline & & Atovaquone resistance for Babesia gibsoni in dogs & 197 \\
\hline \multirow[b]{2}{*}{ cyt $b$} & No available codons & Atovaquone resistance for $P$. berghei in vitro & 124 \\
\hline & Codon 268 & $\begin{array}{l}\text { Atovaquone-Pyrimethamine combined } \\
\text { therapy resistance for } P \text {. falciparum }\end{array}$ & $125-127$ \\
\hline \multirow{8}{*}{$d h f r$} & \multirow{2}{*}{ No available codons } & Atovaquone resistance for $T$. gondii & 183 \\
\hline & & Pyrimethamine resistance for $T$. gondii & 184 \\
\hline & Codon 108 (serine $\rightarrow$ asparagine) & $\begin{array}{l}\text { Pyrimethamine resistance for } P \text {. falciparum } \\
\text { in Brazil, New Guinea \& Tanzania }\end{array}$ & $44-46$ \\
\hline & $\begin{array}{l}\text { Codon } 108 \text { (serine } \rightarrow \text { threonine) } \\
+ \text { Codon } 16 \text { (alanine } \rightarrow \text { valine) }\end{array}$ & Proguanil resistance for $P$. falciparum in Africa & 43,47 \\
\hline & Codons $51,59 \& 108$ & Pyrimethamine resistance for $P$. falciparum in India & 71 \\
\hline & Codons 57, 58, \& 117 & $\begin{array}{l}\text { Fansidar resistance for } P \text {. vivax isolated from } \\
\text { widely separated countries from Asia and Africa }\end{array}$ & 78 \\
\hline & Codons 58, 61 and 117 & Pyrimethamine resistance for $P$. vivax in China & 83 \\
\hline & Codons 36,83 or 245 & Different outcomes in the virulence of $T$. gondii & 202 \\
\hline \multirow{5}{*}{ dhps } & No available codons & Sulfonamide resistance for T. gondii & 185 \\
\hline & Codons $436,437 \& 540$ & Sulphadoxine resistance for $P$. falciparum in India & 71,42 \\
\hline & Codons $540 \& 588$ & Sulphadoxine resistance for $P$. falciparum in Indonesia & 76 \\
\hline & Codons $57,58, \& 117$ & $\begin{array}{l}\text { Sulphadoxine resistance for } P \text {. vivax isolated } \\
\text { from malaria endemic countries }\end{array}$ & 79 \\
\hline & Codon 382 & Chloroquine resistance for $P$. vivax in Brazil & 84 \\
\hline \multicolumn{2}{|c|}{ Ferredoxin gene } & Metronidazole resistance in trichomoniasis & 172 \\
\hline \multicolumn{2}{|l|}{$f p^{2}$} & Artemisinin resistance for $P$. falciparum & 151 \\
\hline \multicolumn{2}{|l|}{ hrp2 } & $\begin{array}{l}\text { False diagnosis in falciparum malaria } \\
\text { using rapid diagnostic tests }\end{array}$ & $212-217$ \\
\hline \multicolumn{2}{|c|}{ HSP70 encoding gene } & Antimonial drugs resistance for Leishmania spp. & 192 \\
\hline \multicolumn{2}{|c|}{ kelch13 } & $\begin{array}{l}\text { Artemisinin resistance for } P \text {. falciparum } \\
\text { in Bangladesh, China \& Myanmar }\end{array}$ & $155-159$ \\
\hline \multirow{3}{*}{$m d r$} & \multirow{2}{*}{ Codon 86} & $\begin{array}{l}\text { Chloroquine, mefloquine \& } \\
\text { halofantrine resistance in vitro }\end{array}$ & $85-89$ \\
\hline & & $\begin{array}{l}\text { Increased sensitivity to Mmefloquine } \\
\& \text { halofantrine in Gambia }\end{array}$ & 97 \\
\hline & Codons $86 \& 1246$ & Chloroquine resistance in India & 90 \\
\hline \multicolumn{2}{|l|}{$m r p 2$} & $\begin{array}{l}\text { Quinolones (chloroquine, piperaquine \& mefloquine) } \\
\text { in vitro }\end{array}$ & 136 \\
\hline
\end{tabular}


PARASITOLOGISTS UNITED JOURNAL

\begin{tabular}{|c|c|c|c|}
\hline \multicolumn{2}{|l|}{$n t r$} & Benznidazole resistance for $T$. cruzi & 201 \\
\hline \multicolumn{2}{|c|}{ Patatin-like protein encoding gene } & $\begin{array}{l}\text { Impaired replication of } T \text {. gondii in activated } \\
\text { macrophages }\end{array}$ & 204 \\
\hline \multicolumn{2}{|l|}{$p g p$} & $\begin{array}{l}\text { Metronidazole resistance in amoebiasis, } \\
\text { giardiasis \& trichomoniasis }\end{array}$ & $168-171$ \\
\hline \multicolumn{2}{|l|}{ pgpa } & Oxyanions resistance for Leishmania spp. & 190 \\
\hline \multicolumn{2}{|c|}{ Proteophosphoglycan encoding gene } & Impairmentof $T$. gondii bradyzoite development & 205 \\
\hline \multicolumn{2}{|l|}{ ptr1 } & Methotrexate resistance for Leishmania spp. & 188 \\
\hline \multicolumn{2}{|c|}{$\begin{array}{l}\text { Regulators of chromosome condensation } \\
1 \text { (RCC1) (encoding gene) }\end{array}$} & Impairment of $T$. gondii growth and virulence & 203 \\
\hline \multicolumn{2}{|c|}{ Transmembrane pellicle protein 1 (TPP1) encoding gene } & Impairment of $T$. gondii virulence and invasion & 206 \\
\hline \multicolumn{2}{|c|}{ Topoisomerase I gene } & $\begin{array}{llll}\text { 3,3'-Diindolylmethane } & \text { (DIM) } & \text { resistance } & \text { for } \\
\text { Leishmania } \text { spp. } & & & \end{array}$ & 191 \\
\hline \multicolumn{2}{|c|}{$\begin{array}{l}\text { Thymidine kinase (TK) encoding gene } \\
\text { Uracil phosphoribosyl transferase (UPRT) encoding gene } \\
\text { Uridine phosphorylase (UP) encoding gene }\end{array}$} & $\begin{array}{l}\text { Pyrimidine analogue } 5 \text {-fluorouracil ( } 5 \text { - } \\
\text { FU) resistance for } L \text {. infantum }\end{array}$ & 194 \\
\hline \multicolumn{4}{|c|}{ Combined gene mutations } \\
\hline \multicolumn{2}{|c|}{$\begin{array}{l}\text { crt }(\text { Codon } 76) \\
+\boldsymbol{m d r}(\text { Codon } 86 \text { or } 1246)\end{array}$} & \multicolumn{2}{|l|}{$\begin{array}{l}\text { Amodiaquine resistance for } P \text {. falciparum } \\
\text { in Burkina Faso, Nigeria \& Colombia }\end{array}$} \\
\hline & $\begin{array}{l}\boldsymbol{c r t}(\text { Codon } 72-76) \\
+\boldsymbol{m d r}(\text { Codon } 86)\end{array}$ & $\begin{array}{l}\text { Chloroquine\& Amodiaquine resistance } \\
\text { for P. falciparum in Angola }\end{array}$ & 115 \\
\hline & $\begin{array}{l}\operatorname{crt}(\text { Codon } 76) \\
+\boldsymbol{m} \boldsymbol{d r}(\text { Codon } 86)\end{array}$ & Chloroquine resistance for $P$. falciparum in India & $116-118$ \\
\hline & No available codons & Chloroquine resistance for $P$. vivax in Ethiopia & 123 \\
\hline & $\begin{array}{l}\text { crt }(\text { Codon } 76) \\
+\boldsymbol{m d r} 1 \text { (Codons } 86 \& 184)\end{array}$ & Increased the severity of falciparum malaria & 207 \\
\hline \multirow[t]{2}{*}{$\begin{array}{l}\text { crt } \\
\text { nhe }\end{array}$} & $\begin{array}{l}\text { crt (Codon } 76) \\
\text { nhe (No available codon) }\end{array}$ & $\begin{array}{l}\text { Reduced quinine susceptibility for } P \text {. falciparum in } \\
\text { Kenya, China \& Viet Nam }\end{array}$ & $131-133$ \\
\hline & $\begin{array}{l}\text { dhfr }(\text { Codons } 51 \text { \& 108) } \\
+ \text { dhps }(\text { Codons } 437 \text { \& 581) }\end{array}$ & $\begin{array}{l}\text { Fansidar resistance for } P \text {. falciparum in Uganda and } \\
\text { Venezuela }\end{array}$ & 54,55 \\
\hline \multirow[t]{2}{*}{$\begin{array}{l}\text { dhfr } \\
\text { dhps }\end{array}$} & $\begin{array}{l}\text { dhfr }(\text { Codons } 51,59 \& 108) \\
+ \text { dhps (Codons } 437 \& \text { \&81) }\end{array}$ & $\begin{array}{l}\text { Fansidar resistance for } P \text {. falciparum in Kenya, } \\
\text { Cameroon, Nigeria, Ethiopia, Ghana, Sri Lanka \& } \\
\text { Tanzania }\end{array}$ & $56-62$ \\
\hline & $\begin{array}{l}\text { dhfr }(\text { Codons } 59 \& 108) \\
+ \text { dhps (Codons } 436 \text { or } 437)\end{array}$ & Fansidar resistance for $P$. falciparum in Iran & 70,79 \\
\hline $\begin{array}{l}\text { dhfr } \\
m d r 2\end{array}$ & $\begin{array}{l}\boldsymbol{d h f} \boldsymbol{r}(\text { Codons } 51 \& 58 \text { or } 108) \\
\boldsymbol{m} \boldsymbol{d r} \mathbf{2}(\text { Codon } 423)\end{array}$ & $\begin{array}{l}\text { Pyrimethamine resistance for } P \text {. falciparum in } \\
\text { Thailand }\end{array}$ & 121 \\
\hline
\end{tabular}

Gene abbreviations: aqp2: Gene controlling aquaglyceroporin transporter; bat1: Gene regulating cytokines production; $\boldsymbol{c g} 2$ : A candidate gene for chloroquine resistance; $\boldsymbol{c r t}$ : Chloroquine resistance transporter gene; $\boldsymbol{c y} \boldsymbol{b}$ : Gene encoding mitochondrial apo-cytochrome b; cyt $\boldsymbol{b}$ : Gene encoding cytochrome b; $\boldsymbol{d} \boldsymbol{h} \boldsymbol{f}$ : Dihydrofolate reductase gene; dhps: Dihydropteroate synthase gene; fp2: Gene encoding cysteine protease falcipain-2; hrp2: Gene encoding histidine-rich protein 2; HSP: Heat shock protein; kelch13: Gene encoding Kelch-domain 13; mdr: Multi-drug resistance-1a gene; mrp2: Multidrug resistance protein-2 gene; $\boldsymbol{n h e}$ : sodium hydrogen exchanger gene; $\boldsymbol{n t r}$ : A mitochondrial gene; pgp: P-glycoprotein-like gene; pgpa P-glycoprotein gene; ptr1: Gene encoding pteridine reductase 1.

References are cited in "Gene mutations and parasitic diseases: Part II: Parasite gene mutations (PUJ 2017; 10: 4-22)". 